This information is current as of April 26, 2023.

\title{
Alterations in the Magnetoencephalography Default Mode Effective Connectivity following Concussion
}

D.D. Reddy, E.M. Davenport, F.F. Yu, B. Wagner, J.E. Urban, C.T. Whitlow, J.D. Stitzel and J.A. Maldjian

AJNR Am J Neuroradiol 2021, 42 (10) 1776-1782

doi: https://doi.org/10.3174/ajnr.A7232

http://www.ajnr.org/content/42/10/1776 


\title{
Alterations in the Magnetoencephalography Default Mode Effective Connectivity following Concussion
}

\author{
(DD.D. Reddy, (DE.M. Davenport, (D)F.F. Yu, (D). Wagner, (D).E. Urban, (D)C.T. Whitlow, (D).D. Stitzel, and (D).A. Maldjian
}

\begin{abstract}
BACKGROUND AND PURPOSE: Magnetoencephalography is sensitive to functional connectivity changes associated with concussion. However, the directional influences between functionally related regions remain unexplored. In this study, we therefore evaluated concussion-related magnetoencephalography-based effective connectivity changes within resting-state default mode network regions.
\end{abstract}

MATERIALS AND METHODS: Resting-state magnetoencephalography was acquired for 8 high school football players with concussion at 3 time points (preseason, postconcussion, postseason), as well as 8 high school football players without concussion and 8 age-matched controls at 2 time points (preseason, postseason). Time-series from the default mode network regions were extracted, and effective connectivity between them was computed for 5 different frequency bands. The default mode network regions were grouped into anterior and posterior default mode networks. The combined posterior-to-anterior and anterior-to-posterior effective connectivity values were averaged to generate 2 sets of values for each subject. The effective connectivity values were compared using a repeated measures ANOVA across time points for the concussed, nonconcussed, and control groups, separately.

RESULTS: A significant increase in posterior-to-anterior effective connectivity from preseason to postconcussion (corrected $P$ value $=.013$ ) and a significant decrease in posterior-to-anterior effective connectivity from postconcussion to postseason (corrected $P$ value $=.028$ ) were observed in the concussed group. Changes in effective connectivity were only significant within the delta band. Anterior-to-posterior connectivity demonstrated no significant change. Effective connectivity in the nonconcussed group and controls did not show significant differences.

CONCLUSIONS: The unidirectional increase in effective connectivity postconcussion may elucidate compensatory processes, invoking use of posterior regions to aid the function of susceptible anterior regions following brain injury. These findings support the potential value of magnetoencephalography in exploring directional changes of the brain network following concussion.

ABBREVIATIONS: $\mathrm{EC}=$ effective connectivity; $\mathrm{EEG}=$ electroencephalography; DMN = default mode network; FC = functional connectivity; GC = Granger causality; $\mathrm{MEG}=$ magnetoencephalography; $\mathrm{mTBI}=$ mild traumatic brain injury

I the United States alone, there are an estimated 1.6-3.8 million cases of sports-related concussions each year. ${ }^{1}$ Depending on severity, concussions can lead to cognitive and other neurologic deficits. ${ }^{2}$ The complications that arise from these injuries, which are of particular concern in the developing pediatric brain,

Received October 6, 2020; accepted after revision May 5, 2021.

From the Department of Radiology (D.D.R., E.M.D., F.F.Y., B.W., J.A.M.), University of Texas Southwestern, Dallas, Texas; and Wake Forest School of Medicine (J.E.U., C.T.W., J.D.S.), Winston-Salem, North Carolina.

Support for this research was provided by National Institutes of Health R01 NS082453 and R01 NS091602 (J.A.M., J.D.S., C.T.W.)

Please address correspondence Divya D. Reddy, MS, 5323 Harry Hines Blvd., Dallas, TX 75390; e-mail: divya.reddy@utsouthwestern.edu

- Indicates open access to non-subscribers at www.ajnr.org

http://dx.doi.org/10.3174/ajnr.A7232 emphasize the need for accurate diagnosis and appropriately tailored management. Traditional practices that base treatment on self-evaluations and neuropsychological tests are limited, given the potential subjectivity of these measurements. ${ }^{3}$ Unfortunately, conventional structural imaging modalities such as CT and MR imaging often lack sufficient sensitivity to detect abnormalities. ${ }^{4}$

Given these limitations, there has been increased attention in the development and use of adjunctive noninvasive tools. It is hypothesized that after a concussion, brain function may be altered through the disruption of functional brain networks. ${ }^{5}$ Noninvasive functional neuroimaging techniques designed to interrogate these brain networks, including magnetoencephalography (MEG), electroencephalography (EEG), and fMRI, are, therefore, well-suited for such an evaluation. ${ }^{3}$ 
fMRI indirectly measures alterations in brain function on the basis of fluctuations in the blood oxygenation level caused by neuronal activity and can be corrupted by neurovascular decoupling. In contrast, MEG bypasses the hemodynamic response entirely by directly measuring the magnetic fields induced by neuronal electrical activity in the brain. ${ }^{6-9}$ While EEG is similarly a more direct measure of neuronal activity than fMRI, the electrical signals are contaminated by interference from the conductive and inductive tissues of the brain, skull, and the scalp. The magnetic fields detected by MEG are not affected by these conductive and inductive effects and thereby maintain better signal purity. ${ }^{6,7}$ Recent advances, including higher sensor density and enhanced source reconstruction techniques, have improved the spatial resolution of MEG relative to EEG and are comparable with those of fMRI. ${ }^{7,10}$ Altogether, its high sensitivity and ability to localize pathologic magnetic signals generated by injured brain tissue, even with minimal changes in neuropsychologic testing, make MEG well-suited to assess changes in brain function related to sports-related concussion., ${ }^{4,6,11}$

Several resting-state MEG-based connectivity analyses have revealed alterations in the brain connectivity profiles of concussed subjects and those with mild traumatic brain injury (mTBI). ${ }^{5,12,13}$ Various analytic approaches including amplitude envelope correlations and phase-locking estimates have been used in the past to derive connectivity metrics. ${ }^{13-15}$ Analysis of functional brain architectures can be separated into functional segregation (the identification of a specific anatomic region with a specific function) and functional integration of many specialized cortical regions supporting a single function. Functional integration can be characterized by temporal correlation metrics, including functional connectivity (FC) or effective connectivity (EC). FC tests for coupling between 2 spatially remote brain regions without considering the underlying dynamic process in which causes precede consequences and thus only partly explains the interaction pattern. ${ }^{16} \mathrm{EC}$, on the other hand, is defined by the causal influence that one brain region exerts over another and provides both correlation and directional information, revealing the flow of activity between 2 brain regions. Because coupling in the brain is both directed and reciprocal, functional integration can, therefore, be better understood with EC. ${ }^{17}$

EC can be estimated using 2 main approaches: 1) modeldriven, such as dynamic causal modeling and structural equation modeling, and 2) data-driven, such as Granger causality (GC). Both methods analyze the temporal properties of signals from 2 brain regions and extract information to compute the directed causal influences between them. In this study, we used GC analysis to measure EC for 2 main reasons: 1) Unlike the structural equation or dynamic causal modeling, GC does not need an a priori hypothesis of the existing theoretic framework or EC structure to estimate the temporal precedence, and 2) because GC is applied to MEG data, its inability to account for the relationship between blood oxygen level-dependent signal and neural activity can be ignored. ${ }^{17,18}$

Although FC disruptions in concussed individuals have been studied using $\mathrm{MEG},{ }^{12-14}$ information regarding the directional connectivity changes are rare and remain largely unexplored in this population. We were, therefore, motivated to investigate EC changes. A suitable brain network for analysis would ideally be sensitive to physical trauma affecting different brain locations. The default mode network (DMN) is one such network, having high metabolic demands and important cognitive functions spanning multiple brain regions, and thus is likely to reflect the impact of such insults on the brain at large. ${ }^{19,20}$ Our objective was to study the differences in the EC within the DMN between anterior and posterior DMN regions in concussed, nonconcussed, and age-matched controls. In addition to early postconcussion changes, we also sought to interrogate the late changes that may reflect recovery. Moreover, we hypothesized that like FC, ${ }^{21} \mathrm{EC}$ would exhibit changes within the DMN after concussion and that these changes would gradually normalize during the course of recovery.

\section{MATERIALS AND METHODS Protocol Summary}

All participants included were part of the imaging Telemetry and Kinematic modeLing (iTAKL) study ${ }^{22}$ approved by the institutional review board at Wake Forest Baptist Medical Center and the University of Texas Southwestern. The study was performed with written informed consent by the parents as well as the participants and was Health Insurance Portability and Accountability Actcompliant. The iTAKL study focused on the effects of subconcussive impacts on the brain of high school football players through an entire football season, including preseason and postseason MR imaging, MEG, and cognitive image acquisitions. As a part of this larger study on subconcussive impacts, players who were diagnosed with concussion during the season received an additional postconcussion MR imaging and MEG imaging.

Eight high school football players with concussion, 8 high school football players without concussion, and 8 age-matched controls were included in this study. The football players were from a local high school football team, and the controls were from local noncontact sports teams (swimming and tennis) with no history of concussion or contact sports. The concussed football players ranged between 15.3 and 17.7 years of age with a mean age of $16.1(\mathrm{SD}=0.7)$ years; the nonconcussed football players ranged from 15.4 to 16.8 years of age with a mean age of $16.2(\mathrm{SD}=0.6)$ years; and the controls ranged from 14.9 to 17.1 years of age with a mean age of $15.7(\mathrm{SD}=0.8)$ years. Controls and the nonconcussed group were \pm 1 month agematched and \pm 0.1 body mass index-matched to the concussed group. All participants in this study were male. If a concussion was suspected by the on-site certified athletic trainer, the player was sent to a sports medicine physician to confirm the diagnosis. The players underwent MR imaging and MEG scans at preseason, postseason, and a clinical diagnosis of concussion (postconcussion). The preseason scans were acquired a few days to 1 month in advance of the first practice session, and the postseason scans were acquired within 1 month of the end of football season. For the postconcussion scans, an interval of 1-8 days postinjury was allowed. All concussed players followed return-to-play protocols and were scanned postseason. The mean time between preseason and postconcussion scans was $69(\mathrm{SD}=6.46)$ days, and the mean time between preseason and postseason scans was 135.2 ( $\mathrm{SD}=23.7)$ days. Controls underwent scans at 2 different 


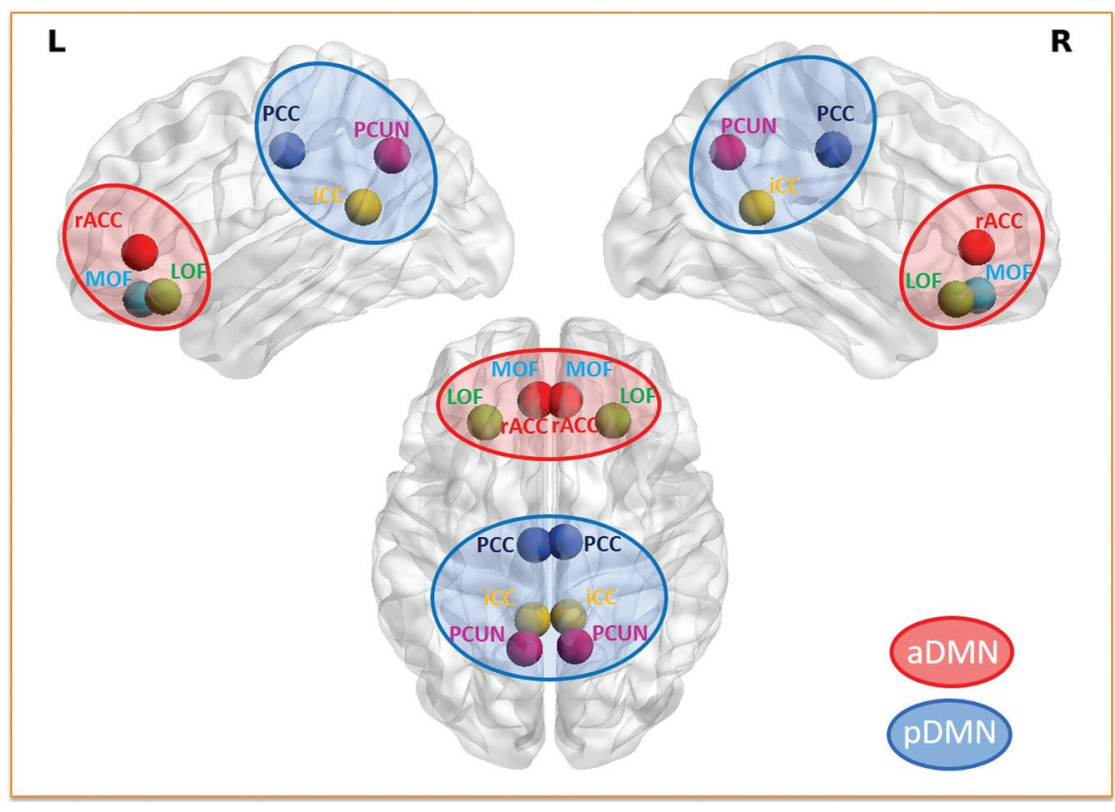

FIG 1. ROIs. DMN regions including the lateral orbitofrontal (LOF), medial orbitofrontal (MOF), and rostral anterior cingulate $(\mathrm{rACC})$ regions grouped into anterior $\mathrm{DMN}$ regions are highlighted in red, and the precuneus (PCUN), posterior cingulate (PCC), and isthmus cingulate (iCC) regions grouped into posterior DMN regions are highlighted in blue. aDMN indicates anterior DMN; pDMN, posterior DMN.

time points approximately 4 months apart, with the mean time between scans being $127.4(\mathrm{SD}=25.2)$ days.

Exclusion criteria included a previous history of concussion, reported neurologic disease, developmental disorders, or psychological conditions. Of the 11 concussed players identified from the iTAKL study, 3 were excluded, including 2 who did not have a pre-/postseason scan and a third who had an abnormal finding on brain MR imaging.

\section{Data Acquisition and Preprocessing}

Resting-state MEG signals sampled at a rate of $1200 \mathrm{~Hz}$ were recorded using a 273-channel radial gradiometer and a 29-channel reference sensor whole-head CTF Omega 2005 MEG system (VSM MedTech) for 8 minutes. Subjects had their eyes open and fixated on a point throughout the data acquisition, and the acquisition bandwidth was set to $0.25-150 \mathrm{~Hz}$. A 3T Magnetom Skyra MR imaging scanner (Siemens) was used to acquire MR imaging data using a 32-channel high-resolution human head/neck coil (Siemens). A structural 3D volumetric T1-weighted MPRAGE sequence with an isotropic resolution of $0.9 \mathrm{~mm}(\mathrm{TR}=1900 \mathrm{~ms}$; $\mathrm{TE}=2.93 \mathrm{~ms} ; \mathrm{TI}=900 \mathrm{~ms}$; flip angle $=9^{\circ} ; 176$ slices) was acquired. Fiducial markers were placed on the subjects' nasion and bilateral preauricular regions to enable the coregistration of MEG data with the MR imaging. The T1 images were normalized to the standard Montreal Neurological Imaging space using the Diffeomorphic Anatomical Registration Through Exponentiated Lie Algebra (DARTEL, part of SPM) toolbox high-dimensional warping and the SPM8 ${ }^{23}$ (http://www.fil.ion. ucl.ac.uk/spm/software/spm12) new segment procedure, as implemented in the VBM8 toolbox ${ }^{23}$ (http://dbm.neuro.uni- jena.de/vbm.html_) and subsequently coregistered with the MEG data.

Acquired MEG data were processed using the Brainstorm ${ }^{24}$ toolbox in Matlab (MathWorks). The MEG signals were baseline-corrected, downsampled to $250 \mathrm{~Hz}$, notch-filtered at $60 \mathrm{~Hz}$, and bandpass-filtered between 1 and $100 \mathrm{~Hz}$. The data were further divided into 5 different frequency bands consisting of delta $(2-4 \mathrm{~Hz})$, theta $(4-8$ $\mathrm{Hz})$, alpha $(8-12 \mathrm{~Hz})$, beta $(12-30 \mathrm{~Hz})$, and gamma $(30-50 \mathrm{~Hz})$. Eye blinks and muscle artifacts were identified and removed using Independent Component Analysis. Noise covariance was calculated using an empty room scan. After preprocessing, time recordings from 60 to 240 seconds (3 minutes) for each subject were used to ensure that stable and quality data were included for the subsequent processing and analysis. A cortex surface head model with overlapping spheres was then constructed for the 3 minutes of clean data for each frequency band. Sources were computed for the MEG data by projecting the signal onto the standard source space model using the whitened and depth-weighted linear-L2 minimum norm estimates algorithm. ${ }^{25}$ Finally, ROIs were extracted for the effective connectivity analysis.

\section{ROI}

The Desikan-Killiany ${ }^{26}$ cortical atlas partitions the whole cortex into 68 regions. Fourteen bilateral cortical DMN regions were used, including lateral orbitofrontal, medial orbitofrontal, rostral anterior cingulate, parahippocampal, isthmus cingulate, posterior cingulate, and precuneus. ${ }^{27}$ For a better understanding of the directionality of connectivity on a large scale, the DMN regions were grouped into anterior and posterior DMN. Anterior DMN included both left and right lateral orbitofrontal, medial orbitofrontal, and rostral anterior cingulate regions, while the posterior DMN included both the left and right isthmus cingulate, posterior cingulate, and precuneus regions as shown in Fig 1. All the regions of the anterior DMN as per the Montreal Neurological Institute coordinates had $\mathrm{Y}>16$ as the centroid of those regions, and the posterior DMN consisted of regions with centroid $\mathrm{Y}<-40$. The parahippocampal region, which did not fall under either the anterior DMN or posterior DMN, was not included in the connectivity analysis. ${ }^{28}$ A mean time-series was extracted from the remaining $12 \mathrm{DMN}$ regions using the Desikan automated parcellation approach, and the EC analysis was then performed between them.

\section{Effective Connectivity and Granger Causality}

EC estimating the strength and directionality of causal relationships between brain regions can be assessed using GC. GC is a 


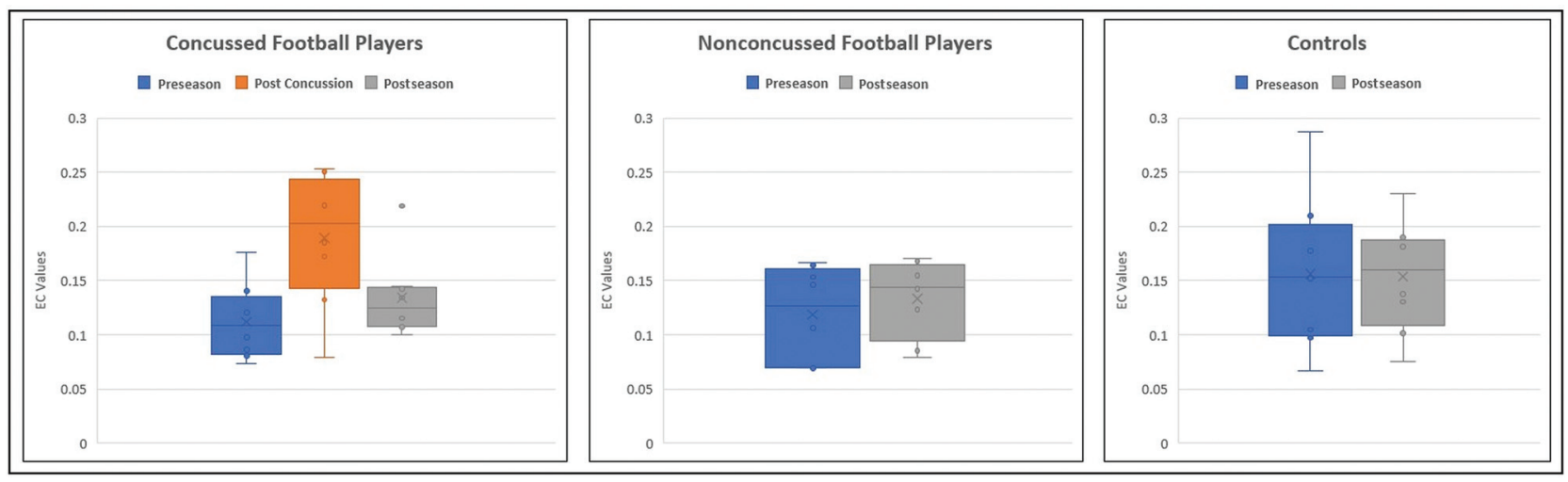

FIG 2. Comparison of effective connectivity value changes across time. EC values at preseason, postconcussion, and postseason for the concussed group (left), EC values at preseason and postseason for the nonconcussed group (center), and EC values at preseason and postseason in controls (right).

statistical approach that measures the extent to which one timeseries signal plays a role in predicting the other. Assuming that the time-series of one ROI is represented by $\mathrm{X}$ and the other ROI is represented by $\mathrm{Y}$, the principle of GC can be defined as $\mathrm{Y}$ causes $\mathrm{X}$ if $\mathrm{Y}$ is able to better predict $\mathrm{X}$ by including all previous information about $\mathrm{Y}$ compared with using previous information of X alone. ${ }^{29}$ Two signals simultaneously acquired and of the same length can be represented as a bivariate linear autoregressive model in the time domain as follows:

$$
\begin{aligned}
X(t) & =\sum j=1: p A 11 X(t-1)+\sum j=1 \\
& : p A 12 Y(t-j)+E 1(t), \\
Y(t) & =\sum j=1: p A 21 X(t-1)+\sum j=1 \\
& : p A 22 Y(t-j)+E 2(t),
\end{aligned}
$$

where $p$ represents the maximum lag in the autoregressive model known as the model order, A comprises the estimated coefficients of the model, and E1 and E2 are the residuals for each time-series. On the basis of this model, if the variance of the E1 prediction error term is reduced by including the $\mathrm{Y}$ term in the first equation, then $\mathrm{Y}$ Granger causes $\mathrm{X}$, and if the $\mathrm{E} 2$ predicted error is reduced with the inclusion of the $\mathrm{X}$ term in the second equation, then it indicates that $\mathrm{X}$ Granger causes $\mathrm{Y}$. The optimum model order was set to a default of 10 . The autoregressive model can be extended to the MEG timeseries data from all the 12 ROIs to generate an $12 \times 12$ asymmetric values matrix, which has different measures for $\mathrm{X}$ causing $\mathrm{Y}$ and $\mathrm{Y}$ causing $X$. The GC will result in a value of zero if there are no causal influences present and as non-negative values otherwise. ${ }^{30}$

\section{Statistical Analysis}

An effective connectivity matrix using the GC model was obtained for each subject for all 5 different frequency bands. The normalized connectivity values for posterior-to-anterior DMN regions and anterior-to-posterior DMN regions were averaged to generate 2 sets of values for each of the 5 frequency bands per subject. The connectivity values between the ROIs within the DMN providing the same region connectivity, ie, posterior-toother posterior and anterior-to-other anterior DMN regions, were not considered for the analysis. A repeated measures ANOVA was used to compare the means across different time points for each combination of frequency band and connectivity orientation within the concussed, nonconcussed, and controls group separately. The resulting $P$ values were adjusted using a Bonferroni multiple comparisons correction. Post hoc pair-wise testing with a Bonferroni correction between time points was performed with only the overall adjusted $P$ value $<.05$. Differences in preseason scans were also compared for all 3 subject groups. All statistical analysis was performed using R Studio (http:// rstudio.org/download/desktop).

\section{RESULTS}

A total of $36 \mathrm{EC}$ values comprising the posterior-to-anterior DMN connectivity for both cerebral hemispheres were averaged. The same process was carried out to obtain the anterior-toposterior DMN EC value for each subject. Repeated measures ANOVA revealed significant differences across different time points for the posterior-to-anterior DMN connectivity within the delta frequency band only in the concussed group. The EC values were plotted across the season for the concussed, nonconcussed, and control groups. Figure 2 is a box-and-whisker plot demonstrating the spread of EC values for all 8 subjects at each time point for the concussed, nonconcussed, and control subjects. The concussed group had an increase in the EC between the pre- and postconcussion scans for all subjects. The increased postconcussion connectivity is followed by a decrease in the connectivity postseason, partially returning to the level of the baseline (preseason) scan. Only the posterior-to-anterior connectivity showed significant differences. Of all the frequency bands, significant EC differences were noted only for the delta band. Also, the spread of EC values for all the subjects in the nonconcussed and control groups did not vary between time points.

\section{Effective Connectivity in Concussed Subjects}

Repeated measures ANOVA was performed for each combination of band and orientation, generating $10 P$ values for the concussed group. Only the delta band showed significant Bonferroni-corrected differences in the posterior-to-anterior EC (corrected $P$ value $=.005$ ). The post hoc tests revealed a 


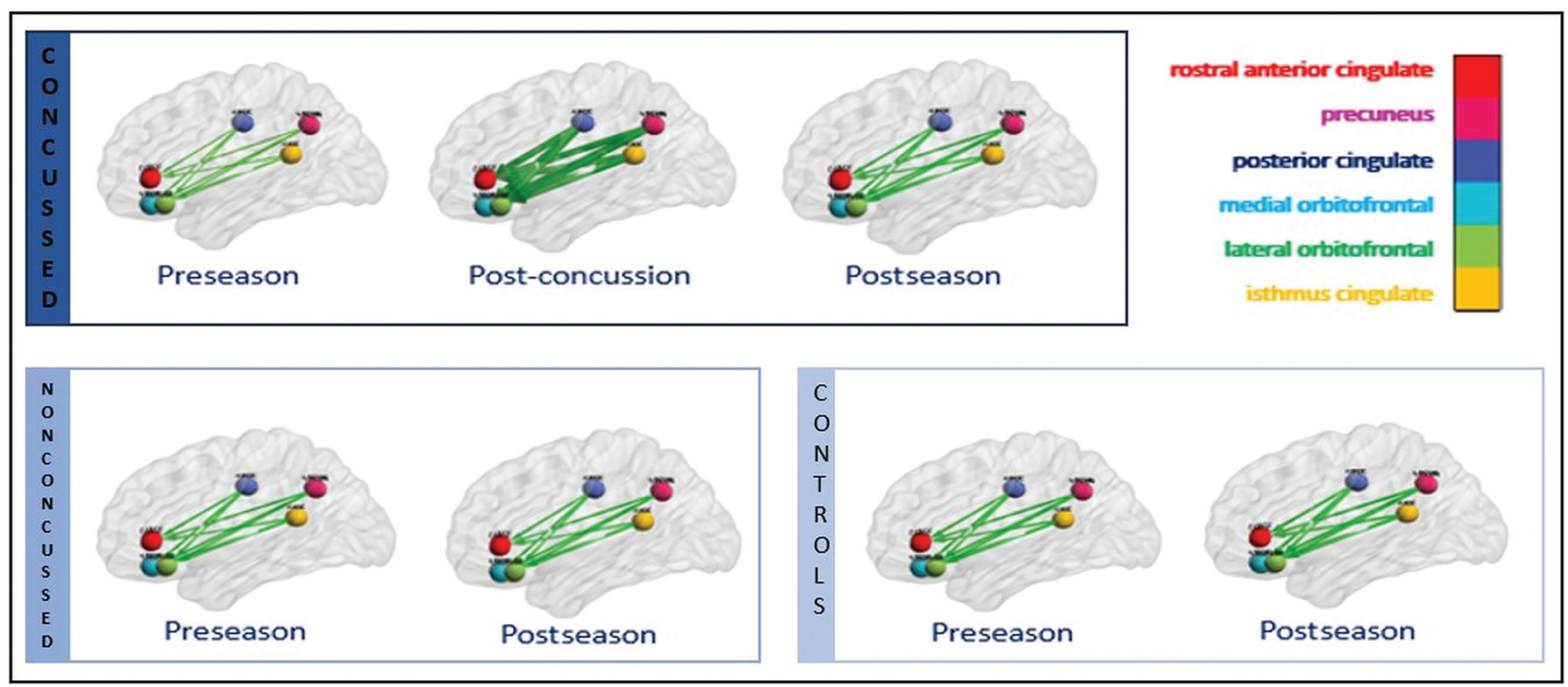

FIG 3. Visual representation of averaged EC across subjects for the concussed (upper row), nonconcussed (lower left), and control (lower right) groups. The concussed group demonstrates a unidirectional increase in EC from preseason to immediately postconcussion $(P=.013)$ and a decrease at postseason scan compared with the postconcussion scan $(P=.028)$. The nonconcussed group and controls reveal no significant difference in EC between preseason and postseason scans.

significant increase in the mean EC from preseason to postconcussion for the concussed players (corrected $P$ value $=.013$ ). A consistent increase in EC was observed for each subject, with the average percentage increase for the group at approximately $8 \%$ followed by a significant decrease in the effective connectivity from postconcussion to the postseason scans (corrected $P$ value $=.028$ ), at approximately $6 \%$ decrease. Despite the decreases at the postseason scan, the effective connectivity remained significantly different compared with the preseason scan (corrected $P$ value $=.028$ ). Figure 3 provides a visual representation of the longitudinal unidirectional increase and decrease of EC throughout the season, averaged across all 8 subjects. Figures 1 and 3 were generated using BrainNet Viewer, a matlab-based network visualization toolbox (www.nitrc.org/projects/bnv/).

Of note, one of the concussed subjects who was not included in the study due to lack of a postseason scan exhibited similar results between the preseason baseline and postconcussion scan, with an increase of $5.9 \%$ in the total posterior-to-anterior connectivity.

\section{Effective Connectivity in Nonconcussed Subjects and Controls}

Repeated measures ANOVA revealed no significant EC difference between preseason and postseason scans for the nonconcussed football players ( $P$ value for delta $=.392$ ). The averaged network for the controls did not show any major changes in the connectivity strength between the pre- and postseason scans either ( $P$ value for delta $=.947$ ) for any combination of band and orientation.

The preseason scans compared across different subject groups using independent 2-sample $t$ tests demonstrated no significant changes between the concussed to nonconcussed groups $(P$ value $=.748)$, the nonconcussed group to controls $(P$ value $=$ .289), and the concussed group to controls ( $P$ value $=.095)$.

The Post-Concussion Symptom Inventory survey scores account for symptoms in 20 categories and were available for only 5 of the 8 concussed subjects who were used for the analysis. The average Post-Concussion Symptom Inventory scores were 2.2 for preseason, 27 for postconcussion, and 6.4 for postseason. Although we did not have the post-concussion symptom scores for all our subjects, the correlation between the average EC changes and the average Post-Concussion Symptom Inventory scores across the season had a high $R^{2}$ value of 0.98 .

\section{DISCUSSION}

Alterations in brain FC have been studied in patients with mTBI and concussions ${ }^{13,31,32}$ using graph theory, power spectral density, and correlation analysis. However, these analyses did not use methods for providing both magnitude and directionality information. Here, we set out to explore whether an MEG-based EC method could capture variations in directed connectivity in concussed individuals, nonconcussed individuals, and matched controls. Using GC, we found that EC increased significantly in high school football players immediately after concussion followed by a significant decrease observed at the postseason time point. The EC at the postseason time point remained significantly increased compared with the preseason baseline, suggesting only a partial recovery. Additionally, EC in the DMN changed only in 1 direction, from posterior to anterior, and only affected the delta band. EC showed no significant changes in the nonconcussed individuals (both controls and football players) through the season.

Increased brain connectivity or hyperconnectivity is believed to be a fundamental adaptation of neuronal systems to physical disruptions resulting from brain injury, referred to as network reorganization. ${ }^{33}$ Studies suggest that connectivity increases may represent a compensatory mechanism of the brain in response to the functional impairment caused by axonal injury, invoking the use of more connections to perform the same tasks and resulting in increased synaptic activity measured by MEG. ${ }^{34-36}$ EC increases have also been attributed to the regulation of dysfunctional neural 
systems and compensatory processes in conditions like bipolar disorder $^{37}$ and Alzheimer disease. ${ }^{38}$ In some cases, EC increases have also been associated with improved corticocortical interactions in the damaged hemispheres of patients with stroke, facilitating recovery. ${ }^{39}$ The EC increases in our concussed subjects support the hypothesis of a compensatory mechanism.

Most interesting, significant EC increases were observed only in the posterior-to-anterior direction. Neuropsychological dysfunction affecting executive, cognitive, or motor functions may be a reflection of a predominance of injuries to the frontal and anterior cortices in patients with mTBI. ${ }^{40-42}$ However, posterior regions have also been reported as vulnerable to injuries. ${ }^{43}$ Despite these variations in regions reported as susceptible to injury, functional imaging findings are similar. An fMRI metaanalysis found that anterior brain regions had higher activity than the posterior regions in mTBI, suggesting that the anterior regions are more prone to injury. ${ }^{40}$ Zhou et $\mathrm{al}^{43}$ reported FC decreases within the posterior components and increases in the anterior components of the DMN after mTBI. These findings could account for the unidirectional EC increases from posterior-to-anterior DMN regions, reflecting a compensatory mechanism. As the vulnerable anterior regions are injured postconcussion, the use of other brain regions including the posterior regions to assist in function may result in posterior-to-anterior information flow within the DMN.

Abnormal increases in low-frequency neuronal activity have been long associated with mTBI and can be measured and localized with the help of MEG. ${ }^{411,12,42}$ A handful of animal and human studies have pointed to cortical deafferentation following axonal injury as the main mechanism for delta wave generation after mTBI. ${ }^{4,42}$ Delta waves can be induced by administering atropine in animals. Atropine causes deafferentation by blocking antagonist acetylcholine within the cholinergic pathway. These animal studies have demonstrated that mTBI causes dysregulation of the cholinergic system and chronic reduction of acetylcholine. ${ }^{44,45}$ This result suggests that dysfunction at the synaptic level can cause delta wave generation without cell death. Another explanation for the increase in EC only in the delta band is based on the bistability concept. Bistability is often demonstrated by slow oscillations, which specifically arise in the setting of functional or anatomic disconnection. ${ }^{46}$ These stereotypical slow waves in the delta frequency range result from perturbations to the brain such as concussions or traumatic brain injuries, which may force the intact cortical circuits into a bistable state. Additional studies are needed to delineate the mechanism behind increases in delta activity after mTBI in humans.

Recovery following concussion is often a long and challenging process. Enhancing our understanding of recovery is important to help explain why debilitating symptoms persist in some individuals while resolving in others as well as to aid in personalized patient management. In this study, MEG demonstrated functional abnormalities in the concussed population even at postseason, suggesting that alterations in brain function persisted after athletes were cleared for return to play. Despite significant decreases in connectivity postseason from postconcussion, the connectivity remained significantly higher than the preseason baseline, implying that a longer recovery period may be needed. Whether a portion of these cases go on to ultimately recover to baseline remains to be explored and highlights the need for additional large, long-term studies.

\section{Limitations}

Our study included only male adolescent football players and had a small sample size, limiting the generalizability of our results. Inclusion of orthopedically injured players can address confounding factors like pain or fatigue associated with the alterations in connectivity. The partial normalization implies that the connectivity changes may extend beyond the 3 -month postseason timeframe, necessitating large-scale longitudinal studies to further clarify the course of recovery. Furthermore, analyzing the connectivity relationships, including both intra- and internetwork EC (such as frontoparietal or even task-based networks aside from the DMN), could provide additional information on compensatory pathways and network anatomy. Finally, it would also be worth exploring the relationship between measures of cognitive assessment and structural connectivity, such as with diffusion MR imaging, and FC.

\section{CONCLUSIONS}

GC analysis of MEG data demonstrates that a single episode of concussion can cause observable changes in the EC within the DMN. The EC partially returns toward the baseline level with time. The connectivity changes occur in the delta band, exhibit directionality, and are only significant from posterior to anterior DMN regions. Further large-scale, longitudinal research will be required to delineate the utility of this approach as a biomarker in concussion.

\section{ACKNOWLEDGMENTS}

We thank Dr Yin Xi (Assistant Professor, University of Texas Southwestern Medical Center) for his valuable suggestions and guidance in statistical analysis.

Disclosures: Divya D. Reddy-RELATED: Grant: National Institutes of Health.* Elizabeth M. Davenport-RELATED: Grant: National Institutes of Health*; UNRELATED: Grants/Grants Pending: National Institutes of Health, American Society of Neuroradiology.* Jillian E. Urban-RELATED: Grant: National Institutes of Health funding*; UNRELATED: Patents (Planned, Pending or Issued): 1 patent pending for a Mouthpiece Form Factor to measure head impacts. Joel D. StitzelRELATED: Grant: National Institutes of Health, Comments: National Institutes of Health R01NS082453 and R01NS091602*; UNRELATED: Grants/Grants Pending: National Institutes of Health, Comments: National Institutes of Health R01NS082453 and R01NS091602.* Joseph A. Maldjian-RELATED: Grant: National Institutes of Health grant*; UNRELATED: Consultancy: BioClinica, Comments: independent reader for brain tumor clinical trials. *Money paid to the institution.

\section{REFERENCES}

1. Virji-Babul N, Hilderman CG, Makan N, et al. Changes in functional brain networks following sports-related concussion in adolescents. J Neurotrauma 2014;31:1914-19 CrossRef Medline

2. McCrea M, Guskiewicz KM, Marshall SW, et al. Acute effects and recovery time following concussion in collegiate football players: the NCAA Concussion Study. JAMA 2003;290:2556-63 CrossRef Medline

3. Tormenti M, Krieger D, Puccio AM, et al. Magnetoencephalographic virtual recording: a novel diagnostic tool for concussion. Neurosurgical Focus 2012;33:E9 1-7 CrossRef Medline

4. Huang MX, Theilmann RJ, Robb $A$, et al. Integrated imaging approach with MEG and DTI to detect mild traumatic brain injury 
in military and civilian patients. J Neurotrauma 2009;26:1213-26 CrossRef Medline

5. Zhu DC, Covassin T, Nogle S, et al. A potential biomarker in sportsrelated concussion: brain functional connectivity alteration of the default-mode network measured with longitudinal resting-state fMRI over thirty days. J Neurotrauma 2015;32:327-41CrossRef Medline

6. Fibel K, Cardinale N, Nichols S, et al. Evaluating concussion in sport: a case for magnetoencephalography. $J$ Athletic Enhancement 2014;3:4 CrossRef

7. Brookes MJ, Woolrich M, Luckhoo H, et al. Investigating the electrophysiological basis of resting state networks using magnetoencephalography. Proc Natl Acad Sci U S A 2011;108:16783-88 CrossRef Medline

8. Dale AM, Liu AK, Fischl BR, et al. Dynamic statistical parametric mapping: combining fMRI and MEG for high-resolution imaging of cortical activity. Neuron 2000;26:55-67 CrossRef Medline

9. Schwartz ES, Edgar JC, Gaetz WC, et al. Magnetoencephalography. Pediatr Radiol 2010;40:50-58 CrossRef Medline

10. Maldjian JA, Davenport EM, Whitlow CT. Graph theoretical analysis of resting-state MEG data: Identifying interhemispheric connectivity and the default mode. Neuroimage 2014;96:88-94 CrossRef Medline

11. Lee RR, Huang M. Magnetoencephalography in the Diagnosis of Concussion. In: Concussion. Vol 28. Karger Publishers; 2014:94-111

12. Dunkley B, Da Costa L, Bethune A, et al. Low-frequency connectivity is associated with mild traumatic brain injury. Neuroimage Clin 2015;7:611-21 CrossRef Medline

13. Dimitriadis SI, Zouridakis G, Rezaie R, et al. Functional connectivity changes detected with magnetoencephalography after mild traumatic brain injury. Neuroimage Clin 2015;9:519-31 CrossRef Medline

14. Dunkley BT, Urban K, Da Costa L, et al. Default mode network oscillatory coupling is increased following concussion. Front Neurol 2018;9:280 CrossRef Medline

15. Tarapore PE, Findlay AM, LaHue SC, et al. Resting state magnetoencephalography functional connectivity in traumatic brain injury. $J$ Neurosurg 2013;118:1306-16 CrossRef Medline

16. Friston KJ. Functional and effective connectivity: a review. Brain Connect 2011;1:13-36 CrossRef Medline

17. Tana MG, Sclocco R, Bianchi AM. GMAC: a Matlab toolbox for spectral Granger causality analysis of fMRI data. Comput Biol Med 2012;42:943-56 CrossRef Medline

18. Friston K, Moran R, Seth AK. Analysing connectivity with Granger causality and dynamic causal modelling. Curr Opin Neurobiol 2013;23:172-78 CrossRef Medline

19. Sharp DJ, Beckmann CF, Greenwood R, et al. Default mode network functional and structural connectivity after traumatic brain injury. Brain 2011;134:2233-47 CrossRef Medline

20. Raichle ME, MacLeod AM, Snyder AZ, et al. A default mode of brain function. Proc Natl Acad Sci U S A 2001;98:676-82 CrossRef Medline

21. Bharath $\mathrm{RD}$, Munivenkatappa A, Gohel S, et al. Recovery of resting brain connectivity ensuing mild traumatic brain injury. Front Hum Neurosci 2015;9:513 CrossRef

22. Urban JE, Davenport EM, Golman AJ, et al. Head impact exposure in youth football: high school ages 14 to 18 years and cumulative impact analysis. Ann Biomed Eng 2013;41:2474-87 CrossRef Medline

23. Ashburner J, Friston KJ. Voxel-based morphometry: the methods. Neuroimage 2000;11:805-21 CrossRef Medline

24. Tadel F, Baillet S, Mosher JC, et al. Brainstorm: a user-friendly application for MEG/EEG analysis. Comput Intell Neurosci 2011;2011:879716 CrossRef Medline

25. Hämäläinen MS, Ilmoniemi RJ. Interpreting magnetic fields of the brain: minimum norm estimates. Med Biol Eng Comput 1994;32:3542 CrossRef Medline

26. Desikan RS, Ségonne F, Fischl B, et al. An automated labeling system for subdividing the human cerebral cortex on MRI scans into gyral based regions of interest. Neuroimage 2006;31:968-80 CrossRef Medline
27. Kabbara A, Falou WE, Khalil M, et al. The dynamic functional core network of the human brain at rest. Sci Rep 2017;7:2936 CrossRef Medline

28. Hillebrand A, Tewarie P, Van Dellen E, et al. Direction of information flow in large-scale resting-state networks is frequency-dependent. Proc Natl Acad Sci U S A 2016;113:3867-72 CrossRef Medline

29. Granger CW. Investigating causal relations by econometric models and cross-spectral methods. Econometrica 1969;37:424-38 CrossRef

30. Geweke J. Measurement of linear dependence and feedback between multiple time series. J Am Stat Assoc 1982;77:304-13 CrossRef

31. Li L, Pagnotta MF, Arakaki X, et al. Brain activation profiles in mTBI: Evidence from combined resting-state EEG and MEG activity. Annu Int Conf IEEE Eng Med Biol Soc 2015;2015:6963-66 CrossRef Medline

32. Mayer AR, Mannell MV, Ling J, et al. Functional connectivity in mild traumatic brain injury. Hum Brain Mapp 2011;32:1825-35 CrossRef Medline

33. Hillary FG, Rajtmajer SM, Roman CA, et al. The rich get richer: brain injury elicits hyperconnectivity in core subnetworks. PLoS One 2014;9:e104021 CrossRef Medline

34. Tang CY, Eaves E, Dams-O'Connor K, et al. Diffuse disconnectivity in traumatic brain injury: a resting state fMRI and DTI study. Transl Neurosci 2012;3:9-14 CrossRef Medline

35. Palacios EM, Sala-Llonch R, Junque C, et al. Resting-state functional magnetic resonance imaging activity and connectivity and cognitive outcome in traumatic brain injury. JAMA Neurol 2013;70:84551 CrossRef Medline

36. Hristopulos D, Babul A, Brucar L, et al. Disrupted information flow in resting-state in adolescents with sports related concussion. Front Hum Neurosci 2019;13:419 CrossRef Medline

37. de Almeida JR, Versace A, Mechelli A, et al. Abnormal amygdalaprefrontal effective connectivity to happy faces differentiates bipolar from major depression. Biol Psychiatry 2009;66:451-59 CrossRef Medline

38. Liu Z, Zhang Y, Bai L, et al. Investigation of the effective connectivity of resting state networks in Alzheimer's disease: a functional MRI study combining independent components analysis and multivariate Granger causality analysis. NMR Biomed 2012;25:1311-20 CrossRef Medline

39. Wang L, Zhang J, Zhang Y, et al. Conditional granger causality analysis of effective connectivity during motor imagery and motor execution in stroke patients. BioMed Res Int 2016;2016:3870863 CrossRef Medline

40. Eierud C, Craddock RC, Fletcher S, et al. Neuroimaging after mild traumatic brain injury: review and meta-analysis. Neuroimage Clin 2014;4:283-94 CrossRef Medline

41. Hashimoto K, Abo M. Abnormal regional benzodiazepine receptor uptake in the prefrontal cortex in patients with mild traumatic brain injury. J Rehabil Med 2009;41:661-65 CrossRef Medline

42. Huang MX, Nichols S, Baker DG, et al. Single-subject-based wholebrain MEG slow-wave imaging approach for detecting abnormality in patients with mild traumatic brain injury. Neuroimage Clin 2014;5:109-19 CrossRef Medline

43. Zhou Y, Milham MP, Lui YW, et al. Default-mode network disruption in mild traumatic brain injury. Radiology 2012;265:882-92 CrossRef Medline

44. Gorman LK, Fu K, Hovda DA, et al. Effects of traumatic brain injury on the cholinergic system in the rat. J Neurotrauma 1996;13:457-63 CrossRef Medline

45. Schaul N, Gloor P, Ball G, et al. The electromicrophysiology of delta waves induced by systemic atropine. Brain Res 1978;143:475-86 CrossRef Medline

46. D'Andola M, Rebollo B, Casali AG, et al. Bistability, causality, and complexity in cortical networks: an in vitro perturbational study. Cereb Cortex 2018;28:2233-42 CrossRef Medline 